\section{Relative beginners}

\author{
It's About Time: Understanding Einstein's \\ Relativity \\ N. David Mermin \\ Princeton University Press: 2005.256 pp. \\ $\$ 29.95, £ 18.95$
}

\section{Derek Raine}

Many years ago I was asked to write a book for the 'average teenager' explaining Einstein's theories of relativity. Being naive, and not knowing any average teenagers, I took up the challenge. I thought that I had managed rather well in providing a simple account, until I met adult readers and evening-class students who told me how difficult they had found my book. Well, perhaps the subject is difficult. Hermann Bondi used to say that the public would not understand relativity until there were relativistic toys to play with. Today, computer games could play a role in teaching younger students, and lectures with animations on the web could help a more general audience. But we still turn to explanations of relativity in print.

Relativity can be summed up in a phrase that Einstein used when reflecting on the origin of the theory: "At last it came to me that time itself was suspect." It is the way that this statement is unpacked that distinguishes the many expositions of the subject. David Mermin brings to the task a lifetime of experience in making relativity accessible to the non-specialist student without simplifying more than Einstein's well-known dictum would allow.

It's About Time grew out of an earlier book for high schools and Mermin's lecture notes from a course on relativity for non-scientists at Cornell. The book begins with a thorough consideration of frames of reference. Problems involving the collisions of particles are solved by choosing a frame in which the solution is obvious (usually the centre-of-mass frame). This helps the reader to become familiar with transformations between frames of reference in a newtonian context before using them in a thoroughly unfamiliar one. The discussion also nicely illustrates the principle of relativity.

The author's starting point for the treatment of relativity is the velocity addition law. This was also a key element in Einstein's approach and not, as often treated in textbooks, a simple consequence of the Lorentz transformation. The derivation is obtained by looking at a race between a particle and a photon in various frames of reference, together with the one additional element that characterizes Einstein's relativity: the constancy of the velocity of light.

Mermin gives a central role to a quantitative statement of the relativity of simultaneity. This leads to the slowing down of moving clocks, the contraction of moving rulers, and the formula for the Doppler shift. Typical of the originality of the approach is the intriguing fact that the velocity of light, $c$, is 1 foot per nanosecond, to within about $2 \%$, which dissolves the mystery for the general reader of making $c=1$.

The treatment is complete and involves no more than elementary algebra. Mermin suggests that his readers skip the algebraic manipulation if necessary. This is not the same as skipping the algebraic formulation of the argument, which is key to the exposition. For me the algebraic manipulation is the easy bit: relativity, with all those trains, particles and light beams, always seems to involve arguments that slide away when one tries to reproduce them. This is one reason why relativity professionals rely on space-time diagrams.

The chapter on space-time diagrams is perhaps the most important in the book. All the previous results, together with the invariance of the interval, are re-derived from this point of view, but in a way that avoids analytical geometry and uses only geometrical reasoning (essentially, similar triangles).

As the author says, one cannot write a book on relativity without including a chapter on $E=m c^{2}$. Mermin treats this by means of modifications to the conservation laws for momentum, as required by the relativistic addition law for velocities. The approach is quite technical, and I think that the more traditional thought experiment of a light gun in a railway carriage provides more insight.

The final chapter contains a discussion of the reality of the Lorentz contraction (and time dilation). What causes a rod to contract from the point of view of the moving observer, or the lifetime of a muon to increase, when nothing happens to either in its rest frame? We tend to dismiss this as a question of (spacetime) geometry, not physical causality, despite the fact that Einstein himself returned on several occasions to the dynamic origin of the contraction. Mermin's condusion is that one can seek the answer in the equations of quantum electrodynamics, which are entirely consistent with the kinematic results. Is this purely a matter of taste? Any valid calculation of the length of a rod using any Lorentz invariant theory will give a result in accord with relativity - but does that mean that the calculation gives the cause of the contraction?

Mermin describes his primary audience as non-scientists, but also hopes to address undergraduate and graduate students who might find a few interesting things here. I think the problem for this secondary audience is that there are too many words, and for the general reader, too many equations. That is a pity, because this is a book full of insight with an engaging style. I recommend it to anyone who has to teach the subject to either audience: it's a brilliant basis for a set of lecture notes. Derek Raine is in the Department of Physics and Astronomy, University of Leicester,

Leicester LE1 7RH, UK.

\title{
Little wonder
}

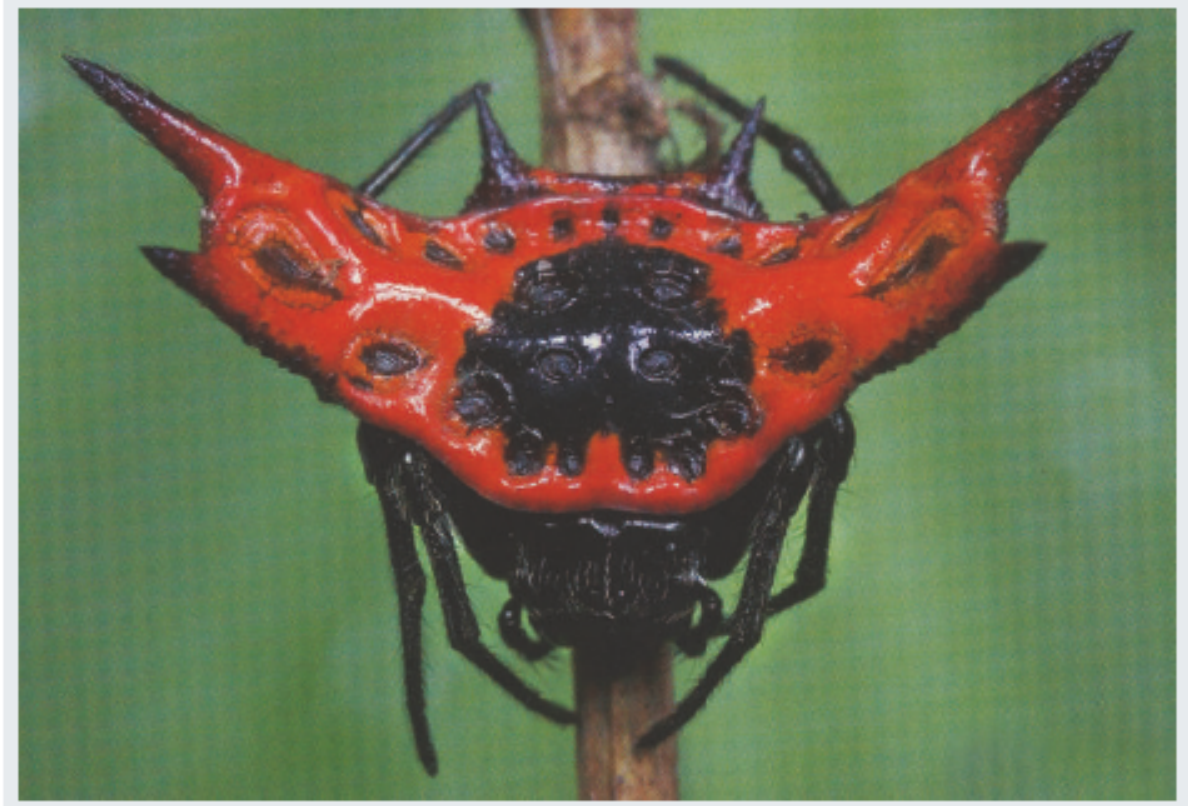

As a conservation biologist, Piotr Naskrecki spendshis time observing some of the smaller animals that inhabit threatened biodiversity hotspots around the globe. Also an accomplished photographer, Naskrecki now shares his images of these tiny creatures, like this armoured spider from the Solomon Islands, in his book The Smaller Majority (Harvard University Press, \$35, £21.95). This beautiful book is a welcome reminder that it is not just the larger, furry creatures that are under threat from habitat destruction. 